\title{
GELFAND DUALITIES OVER TOPOLOGICAL FIELDS
}

\author{
BRIAN J. DAY \\ (Received 26 November 1980) \\ Communicated by R. H. Street
}

\begin{abstract}
Abetract
The spectral duality theory of H.-E. Porst and M. B. Wischnewsky is examined in more generality, and examples based on topological fields are described.
\end{abstract}

1980 Mathematics subject classification (Amer. Math. Soc.): 18 A 40, 18 B 30, 18 D 20.

\section{Introduction}

A spectral duality generated by "topological" algebras is generally taken to mean a Gelfand-type duality between certain function-space algebras and their corresponding spectral spaces. In recent times a general approach to such dualities has been formulated by H.-E. Porst and M. B. Wischnewsky [8] and some important examples of this theory have been supplied by S. S. Hong and L. D. Nel [5]. This framework depends on the notion of a topological closed category and this notion would seem to be fundamental from a practical point of view.

However, it turns out that if the theory of $\mathscr{V}$-enriched epireflective hulls is used then a theory of spectral duality can be formulated over any complete symmetric monoidal closed category $\mathfrak{W}$; this we do in Section 1 . While instances of "non-topological" dualities may be less obvious (functor-category examples do exist), the more general approach brings out the essence of the duality while simplifying the formulation.

The examples produced in this article (in Sections 2, 3, and 4) are, in fact, based on algebras of continuous functions into a topological ring $K$ where $K$ is,

- Copyright Australian Mathematical Society 1982 
algebraically, a field. These yield generalisations of the Gelfand and Stone dualities.

The unexplained notation and terminology of this article are reasonably standard.

\section{Spectral duality; a general theory}

Let $\mathscr{V}=\left(V_{0}, I, \otimes,[-,-], \ldots\right)$ be a complete symmetric monoidal closed base category and suppose that all categorical concepts introduced in this section are relative to this $\mathfrak{V}$. As part of completeness, let $\mathfrak{V}$ admit canonical (epi; strong-mono)-factorisations and also the intersection of any class of strong monomorphisms with a common codomain.

Let $\mathcal{C}$ be a category (enriched over $\mathfrak{W}$ ) which admits canonical (epi; strongmono)-factorisations, equalisers of pairs, and the intersection of any class of strong monomorphisms with a common domain. Moreover, let $K$ be a fixed object of $\mathcal{C}$ such that all the cotensor powers (that is, exponents) of the form $[X, K], X \in \mathfrak{V}$, exist in $\mathcal{C}$.

THEOREM 1.1. There exists a full reflective subcategory $\mathcal{C}$ " of $\mathcal{C}$ such that $K$ strongly $\checkmark$-cogenerates $e "$.

Proof. The category $C^{\prime \prime}$ is simply the ( $V$-enriched) epireflective hull of $K$ in $\mathcal{C}^{\prime}$, where $\mathcal{C}^{\prime}$ is the ('V-enriched) epireflective hull of $K$ in $\mathcal{C}$. The only difficulty is with the existence of canonical (epi; strong-mono)-factorisations in $\mathcal{C}^{\prime}$, but this follows from G. M. Kelly [7, Proposition 4.5(b)] together with the completeness hypotheses on $\mathcal{C}$ itself.

COROLLARY 1.2. The functor $S: \mathcal{C}^{\prime \prime} \rightarrow$ qop, which sends $C$ to $\mathcal{C}(C, K)$, reflects isomorphisms.

We next seek an isomorphism-reflecting adjoint to $S$. This adjoint will essentially be given by suitably restricting the $K$-cotensoring functor $T=[-, K]$ : qop $\rightarrow \mathcal{C}^{\prime \prime}$.

Firstly, suppose that |?|: $\mathcal{C} \rightarrow \mathscr{B}$ is a functor, into a complete category $\mathscr{B}$, such that $|[X, K]| \approx[X,|K|]$ for all $X \in \mathfrak{V}$. An object $X$ of $\mathscr{V}$ is said to be $K$-spectral, with respect to this functor, if there exists an equaliser presentation of the following form in $\mathfrak{T}$ :

$$
X \rightarrow \mathscr{B}(B,|K|) \rightrightarrows \mathscr{B}\left(B^{\prime},|K|\right) ; \quad B, B^{\prime} \in \mathscr{B} .
$$


Let Spec denote the full subcategory of $\mathfrak{V}$ consisting of the $K$-spectral objects of $\mathcal{W}$, and let us call $\mathcal{C} K$-algebraic over $\mathscr{B}$ if each object of the form $\mathcal{C}(C, K)$, $C \in \mathcal{C}$, is $K$-spectral.

THEOREM 1.3. If $C$ is $K$-algebraic over $\mathscr{B}$ then there exists an induced adjunction

$$
(\eta, \varepsilon): T \rightarrow S: \mathcal{C}^{\prime \prime} \rightarrow \operatorname{Spec}^{\text {op }}
$$

in which $T$ also reflects isomorphisms.

Proof. It is clear that the functor $\mathrm{H}: \operatorname{Spec}^{\text {op }} \rightarrow \mathscr{B}$, which maps $X$ to $[X,|K|]$, reflects isomorphisms since $H$ has an adjoint $L$ given by $L(B)=\mathscr{B}(B,|K|)$ and the unit $X \rightarrow \mathscr{B}([X,|K|],|K|)$ of this adjunction is a strong monomorphism in Spec:

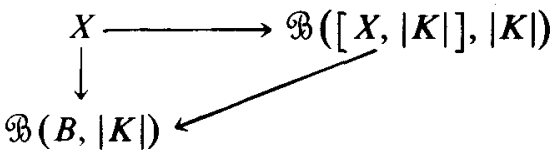

Thus, since $H(X)=[X,|K|] \cong|[X, K]|=|T X|$, we deduce that $T$ reflects isomorphisms.

Now consider the triangle equalities for $T \rightarrow S$.
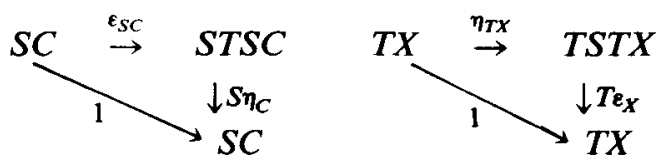

CoROLlaRy 1.4. There exists a spectral duality $\mathcal{C}^{\prime \prime} \simeq \operatorname{Spec}^{\text {op }}$ iff $\varepsilon S$ or $\eta T$ is an epimorphism.

In the examples already constructed by E. Binz [1] and S. S. Hong and L. D. Nel [5], the transformation $\varepsilon_{X}$ is verified to be an epimorphism for all $X \in \mathcal{V}$. This, of course, is a much stronger hypothesis than is needed and leads to the following result.

THEOREM 1.5. If $\varepsilon_{X}$ is an epimorphism for all $X \in \mathbb{V}$ then the inclusion Spec $\subset \mathfrak{V}$ is epireflective and Spec consists of the $K$-embeddable objects of $\mathfrak{V}$.

Here, by a $K$-embeddable object of $\mathfrak{V}$, we mean an object $X$ of $\mathfrak{V}$ which admits a strong $\mathfrak{T}$-embedding into an object of the form $\mathscr{B}(B,|K|)$ for some $B \in \mathscr{B}$. If $\mathscr{B}$ is $\mathfrak{V}$-tensored then the category of $K$-embeddable objects has many nice properties (see [5]). 


\section{Pointwise convergence and $K$-algebras}

Let $\mathcal{V}=($ Top, $1, \otimes,[-,-], \ldots)$ be the category of all topological spaces and continuous maps with the structure of pointwise convergence on the hom-sets. This well-known structure on Top will provide our base category.

Let $\mathscr{B}=\mathcal{V}$ and let $\mathcal{C}$ be the category of topological unital $K$-algebras where $K$ is a topological ring which is algebraically a field and which contains an open set not the whole of $K$. Since a $K$-algebra can be viewed as an action of the form

$$
\sigma_{C}: \sum_{n} \Omega(n) \times C^{n} \rightarrow C,
$$

where $\Omega: \mathbf{N} \rightarrow$ Top is defined in an obvious manner, we have a derived pointwise action

$$
\sigma_{C}: \sum_{n} \Omega(n) \otimes\left(\otimes^{n} C\right) \rightarrow C
$$

which enables us to $\mathfrak{V}$-enrich $\mathcal{C}$ by equalisers of the form:

$$
\mathcal{C}(C, D) \rightarrow[C, D] \rightrightarrows\left[\Sigma \Omega(n) \otimes\left(\otimes^{n} C\right), D\right]
$$

Thus $\mathcal{C}$ is $K$-algebraic over $\mathcal{V}$ and is clearly $\mathcal{V}$-cotensored relative to componentwise structure.

By a standard topological argument (see the first part of the proof of $\mathrm{S}$. Kaplan [6, Theorem 1]) we have that any $\mathcal{C}$-morphism $f:[X, K] \rightarrow K$ gives rise to the following situation

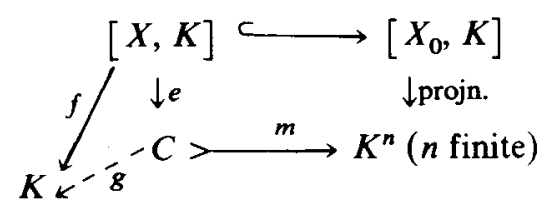

where $X_{0}$ denotes the discrete space on $X$ and $m$ is a continuous injection. In order to deduce that the factorisation of $f$ as ge is such that $g$ lifts along $m$, first note that, by standard structure theory of algebras, the underlying algebra of $C$ is of the form $K^{l}$ ( $l$ finite). Thus $m$ exhausts all projections $K^{l} \rightarrow K$ and so $g$ lifts to a projection which is, of course, continuous.

The conclusion is that $f$ is an evaluation-at- $x$ morphism for some $x \in X$ so $\varepsilon$ : $X \rightarrow \mathcal{C}([X, K], K)$ is a surjection for all topological spaces $X$. By the general theory, this implies the existence of a duality between function-algebras of the form $[X, K]$ and spectral spaces, the latter being those topological spaces which admit an embedding in some $[B,|K|], B \in$ Top. 


\section{Finite discrete fields}

Let $\mathfrak{V}=\left(\mathcal{V}_{0}, 1, \times,[-,-], \ldots\right)$ be the cartesian closed category of all topological $k$-spaces, and let $\mathscr{B}=\mathfrak{V}$. Let $K$ be a finite discrete field, and let $\mathcal{C}$ be the category of unital $K$-algebras in $\mathscr{V}$. It is shown directly that $\varepsilon_{X}$ is a surjection for all $X \in \mathcal{V}$; that is, each $\mathcal{C}$-morphism $f:[X, K] \rightarrow K$ is evaluation-at- $x$ for some point $x \in X$.

Consider the following pushout diagram in the category of ordinary unital rings, where $X_{0}$ denotes the discrete space associated to $X$ :

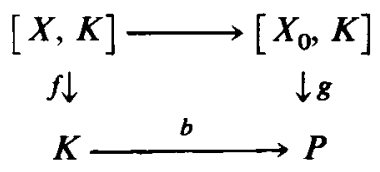

Then $b$ is an injection by W. H. Cornish [2, Theorem 1.6] since all the rings are regular. Give $P$ the quotient topology from $\left[X_{0}, K\right]$, then $g$ is an open mapping. Let $I=\operatorname{ker} g$ and let $Y \subseteq X_{0}$ denote the subset of $X_{0}$ for which there is a non-zero entry of $I$ in the $y \in Y$ coordinate position of $\left[X_{0}, K\right]$. Then $Y \cdot K<I$ $<[Y, K]$ where $Y \cdot K$ denotes the coproduct of $Y$ copies of $K$. Let $\bar{K}$ denote the complement of 0 in $K$ and let $A$ denote the complement of $[Y, \bar{K}]$ in $[Y, K]$. Then $0 \in A \subseteq Y \cdot K \leqslant I$ where $A$ is open by Tychonoff, so $[Y, K] / I$ is discrete. Thus $P \cong([Y, K] / I) \times\left[X_{0}-Y, K\right]$ is a non-zero product of copies of $K$ since $([Y, K] / I)$ is a quotient $K$-algebra of $K^{n}$ ( $n$ finite); see B. J. Day [3]. This means that $b$ splits, and this concludes the proof.

The final result is a duality somewhat analogous to the classical Stone duality for $K=\mathbf{Z}_{2}$. Note that a similar argument can be used to give rise to dualities over finite discrete fields in many "convenient" closed categories.

\section{An application of the Gelfand-Naimark theorem}

Let $\mathcal{V}=(\mathscr{K}, 1, \times,[-,-], \ldots)$ be the cartesian closed category of compactly generated limitspaces; a limitspace $X$ is said to be compactly generated if there exists a small set $\left\{P_{\lambda} ; \lambda \in \Lambda\right\}$ of compact Hausdorff spaces and a limitspace quotient map $\Sigma P_{\lambda} \rightarrow X$. The main example of a $\mathcal{K}$-space is a locally compact hausdorff space (see B. J. Day [4, Section 3]). It is also shown in [4] that there is a cartesian closed $\mathcal{K}$-ification functor $W: \mathscr{B} \rightarrow \mathcal{K}$ where $\mathscr{B}=(\lim , 1, \times$, $(-,-), \ldots)$ is the cartesian closed category of all limitspaces and $[X, Y]=$ $W(X, Y)$ for all $X, Y \in \mathscr{K}$. 
Let $K=\mathbf{C}$, the complex field, and let $\mathcal{C}$ be the $\mathscr{B}$-category of all unital $\mathrm{C}$-algebras in $\mathscr{B}$, regarded as a $\mathscr{K}$-category under the action of $W: \mathfrak{B} \rightarrow \mathscr{K}$. Then $\mathcal{C}$, being monadic over $\mathscr{B}$, is $\mathbf{C}$-algebraic over $\mathscr{B}$ and $\mathcal{C}$ is $\mathbf{C}$-cotensored as a $\mathscr{K}$-category.

Suppose $X \in \mathscr{K}$ and let $\Sigma P_{\lambda} \rightarrow X$ be a presentation of $X$. If $f:[X, \mathrm{C}] \rightarrow \mathrm{C}$ is a $\mathcal{C}$-morphism then it can be extended along the resultant embedding $[X, \mathrm{C}]<$ $\Pi\left[P_{\lambda}, \mathrm{C}\right]$ by the proof of $\mathrm{S}$. Kaplan [6, Theorem 1] coupled with the classical Gelfand-Naimark theorem. In the process, it is seen that the canonical morphism

$$
\operatorname{colim}_{\delta \in \Delta} \mathcal{C}\left(\left[Q_{\delta}, \mathbf{C}\right], \mathbf{C}\right) \rightarrow \mathcal{C}\left(\Pi_{\lambda \in \Lambda}\left[P_{\lambda}, \mathbf{C}\right], \mathbf{C}\right)
$$

is a surjection, where $\Delta$ is the set of all finite subsets of $\Lambda$ and $Q_{\delta}=\Sigma_{\lambda \in \delta} P_{\lambda}$. Thus we consider the diagram:

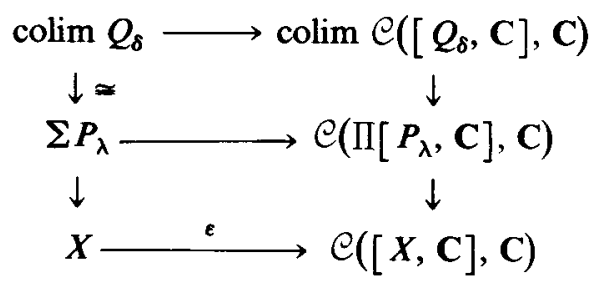

Here the top arrow is a surjection by part of the classical Gelfand duality. Since $\varepsilon_{X}$ is then a surjection for all $X \in \mathcal{K}$, we obtain a spectral duality between function algebras $[X, \mathbf{C}], X \in \mathscr{K}$, and $\mathbf{C}$-embeddable objects $X \in \mathcal{K}$ for which there is an inclusion $X \hookrightarrow W(B, C), B \in \mathscr{B}$.

\section{References}

[1] E. Binz, Continuous convergence on $C(X)$, Lecture Notes in Mathematics 469 (SpringerVerlag, Berlin, Heidelberg, New York, 1975).

[2] W. H. Cornish, 'Amalgamating commutative regular rings', Comment. Math. Univ. Carolinae, 18-3 (1977), 423-436.

[3] B. J. Day, 'Note on duality of Kelleyspace products', Bull. Austral. Math. Soc. 19 (1978), 273-275.

[4] , 'An extension of Pontryagin duality', Bull. Austral. Math. Soc. 19 (1978), 445-456.

[5] S. S. Hong and L. D. Nel, 'Duality theorems for algebras in convenient categories', Math. $Z$. 166 (1979), 131-136.

[6] S. Kaplan, 'Extensions of the Pontryagin duality II: Direct and inverse sequences', Duke Math. J. 17 (1950), 419-435. 
[7] G. M. Kelly, 'Monomorphisms, epimorphisms, and pull-backs', J. Austral. Math. Soc. 9 (1969), 124-142.

[8] H.-E. Porst and M. B. Wischnewsky, 'Every topological category is convenient for Gelfand duality', Manuscripta Math. 25 (1978), 169-204.

School of Mathematics and Physics

Macquarie University

North Ryde, NSW 2113

Australia 